\title{
Risk stratification based on screening history: the NELSON lung cancer screening study
}

\author{
Uraujh Yousaf-Khan, ${ }^{1}$ Carlijn van der Aalst, ${ }^{1}$ Pim A de Jong, ${ }^{2}$ Marjolein Heuvelmans, ${ }^{3}$ \\ Ernst Scholten, ${ }^{2,4}$ Joan Walter, ${ }^{3}$ Kristiaan Nackaerts, ${ }^{5}$ Harry Groen, ${ }^{6}$ \\ Rozemarijn Vliegenthart, ${ }^{3}$ Kevin ten Haaf, ${ }^{1}$ Matthijs Oudkerk, ${ }^{3}$ Harry de Koning ${ }^{1}$
}

\begin{abstract}
- Additional material is published online only. To view please visit the journal online (http://dx.doi.org/10.1136/ thoraxjnl-2016-209892).
\end{abstract}

For numbered affiliations see end of article.

\section{Correspondence to}

Uraujh Yousaf-Khan, Erasmus Medical Center, University Medical Center Rotterdam, Department of Public Health, Room Na 22-09, PO Box 2040, Rotterdam 3000 CA, The Netherlands;

a.yousaf@erasmusmc.nl

Received 20 December 2016 Revised 23 February 2017

Accepted 9 March 2017 Published Online First 30 March 2017

\section{Linked}

- http://dx.doi.org/10.1136/ thoraxjnl-2017-210268

\section{CrossMark}

To cite: Yousaf-Khan U, van der Aalst $C$, de Jong PA, et al. Thorax 2017;72:819-824.

\section{ABSTRACT}

Background Debate about the optimal lung cancer screening strategy is ongoing. In this study, previous screening history of the Dutch-Belgian Lung Cancer Screening trial (NELSON) is investigated on if it predicts the screening outcome (test result and lung cancer risk) of the final screening round.

Methods 15792 participants were randomised (1:1) of which 7900 randomised into a screening group. CT screening took place at baseline, and after 1,2 and 2.5 years. Initially, three screening outcomes were possible: negative, indeterminate or positive scan result. Probability for screening outcome in the fourth round was calculated for subgroups of participants.

Results Based on results of the first three rounds, three subgroups were identified: (1) those with exclusively negative results $(n=3856 ; 73.0 \%)$; $(2)$ those with $\geq 1$ indeterminate result, but never a positive result $(n=1342 ; 25.5 \%)$; and (3) with $\geq 1$ positive result $(n=81 ; 1.5 \%)$. Group 1 had the highest probability for having a negative scan result in round $4(97.2 \%$ vs $94.8 \%$ and $90.1 \%$, respectively, $p<0.001)$, and the lowest risk for detecting lung cancer in round 4 ( $0.6 \%$ vs $1.6 \%, p=0.001)$. 'Smoked pack-years' and 'screening history' significantly predicted the fourth round test result. The third round results implied that the risk for detecting lung cancer (after an interval of 2.5 years) was $0.6 \%$ for those with negative results compared with $3.7 \%$ of those with indeterminate results.

Conclusions Previous CT lung cancer screening results provides an opportunity for further risk stratifications of those who undergo lung cancer screening.

Trial registration number Results, ISRCTN63545820.

\section{INTRODUCTION}

Lung cancer is the leading cause of cancer-related death worldwide. ${ }^{1}$ Lung cancer is often diagnosed at an advanced disease stage and occurs increasingly among former smokers. ${ }^{2}$ This underlines the need for preventive measures. Since 2013, lung cancer screening has been recommended by the United States Preventive Services Task Force. ${ }^{3} 4$ People aged 55 through 80, who have smoked at least 30 pack-years, and currently smoke or have quit smoking within the past 15 years are invited for an annual low-dose CT (LDCT) examination in the USA. However, debate about the optimal screening strategy (eg, the optimal screening interval) is still ongoing. ${ }^{5-9}$

\section{Key messages}

What is the key question?

- If screening history could be used as a risk stratification tool to optimise the screening strategy for lung cancer screening.

\section{What is the bottom line?}

- Previous screening history predicts the screening outcome (screening test result and lung cancer detection risk) for subgroups of NELSON participants.

\section{Why read on?}

- The Dutch-Belgian Lung Cancer Screening trial (NELSON) is the largest European randomised lung cancer screening trial which is sufficiently powered, and it provides an unique opportunity to investigate different screening intervals in one screening group among the lung cancer screening trials.

Currently, lung cancer screening is not implemented in Europe. The main reason is that none of the (underpowered) European lung cancer screening trials have shown a mortality reduction so far. ${ }^{10-13}$ However, Europe's largest trial the DutchBelgian lung cancer screening trial (NELSON) is now in its final follow-up phase and is sufficiently powered to detect a lung cancer mortality reduction of at least 25\%. ${ }^{14-16}$ Main differences between NELSON and the National Lung Screening Trial (NLST) are as follows: (1) the age of the selected subjects (50-74 vs 55-74 years), (2) the use of increasing screening intervals versus annual screening, (3) volumetric-based nodule management versus a diameter-based nodule management and (4) a control group with no screening versus a control group screened with annual chest radiography. The differences in screening interval enable to investigate the optimal screening strategy for lung cancer screening to reduce the probability on potential harms (eg, false-positive examinations leading to unnecessary (non)-invasive diagnostic procedures) for those without lung cancer. ${ }^{4} 1718$ The NLST showed that lung cancer risk and mortality benefit vary within the screened population: the largest mortality benefit was achieved in the subgroup with the highest risk for developing lung 
cancer. ${ }^{4}$ The ratio between benefits and harms of lung cancer screening could be improved by more precisely identifying a high-risk population for developing lung cancer ${ }^{19-21}$ and by risk stratification of subjects based on the individual's screening history (eg, previous screening outcome or presence of a nodule). ${ }^{6}{ }^{22-24}$ Recently, the NLST showed that participants with a negative screening result at baseline have a lower lung cancer detection risk at subsequent screening rounds compared with all screened participants and therefore may not need annual lung cancer screening. ${ }^{7}$ In line with this, the baseline scan in the NELSON trial allowed the identification of three subgroups with different risks for detecting lung cancer in the second and third screening rounds. ${ }^{23}$ Since all NELSON screening rounds with different screening intervals (with a unique 2.5-year interval) have been completed, this study aims to investigate whether NELSON subgroups with different risks for detecting lung cancer can be identified based on their previous screening history. This information might be useful for further risk stratification of subjects who undergo lung cancer screening.

\section{METHODS}

\section{NELSON trial}

In brief, the NELSON trial is a randomised-controlled, population-based lung cancer screening trial. The primary aim is to the investigate whether LDCT screening of high-risk subjects for developing lung cancer can lead to a reduction of lung cancer mortality by $\geq 25 \%$ compared with no screening at 10 years of follow-up. ${ }^{15}$ High-risk subjects, mainly males, were defined as aged between 50 and 74 years, who had smoked at least 15 cigarettes/day for $\geq 25$ years or 10 cigarettes/day for $\geq 30$ years, and were still smoking or had quit $<10$ years ago. ${ }^{14}$ Initially, 15822 participants were randomised (1:1). However, through linkages with the national cancer registries and death registries of the Netherlands and Belgium (Statistics Netherlands and the Flemish Agency for Care and Health, respectively), it appeared that 30 participants $(15$ screening group and 15 control group participants) died before randomisation and should therefore be ruled out from further analysis. After this correction, 15792 participants were randomised (1:1) into a CT screening group (7900) and into a control group $(n=7892)$. Screening took place at baseline, after 1 year, 3 years and 5.5 years. The control group received usual care (no screening). The baseline screening round was conducted from January 2004 through December 2006, and the final screening round was conducted from November 2009 through March 2012.

\section{Study participants}

For the risk stratifications based on the regular scan results of the first three screening rounds, only participants who attended both one of the first three regular screening rounds and round 4 were included $(n=5279)$. For the risk stratification based on the results of the third screening round alone, participants who attended both the third and fourth rounds were included $(\mathrm{n}=5268)$. To compare participants with and without a screening-detected lung cancer across all the screening rounds, all screened participants were included $(n=7582)$.

\section{Screening procedures, outcomes and the nodule management protocol}

Screening was performed using 16-detector CT scanners in low-dose setting at four screening sites (University Medical Center Groningen, University Medical Center Utrecht, Kennemer Gasthuis Haarlem in the Netherlands and University
Hospital Gasthuisberg Leuven in Belgium). ${ }^{25}$ More detailed descriptions of the equipment, the execution of the screening examination and the nodule management protocol have been published previously. ${ }^{15}{ }^{23}{ }^{25-27}$ In short, screening could lead to the following test results: (1) negative: no nodule, newly detected nodule with a volume of $<50 \mathrm{~mm}^{3}$ or previously detected nodule with a growth (change in volume between scans) of $<25 \%$ or $\geq 25 \%$ but with a volume doubling time (VDT) of $>600$ days; (2) indeterminate: newly detected nodule with a volume of $50-500 \mathrm{~mm}^{3}$ or previously detected nodule with a VDT of 400-600 days; or (3) positive: newly detected nodule with a volume of $>500 \mathrm{~mm}^{3}$ or previously detected nodule with a VDT of $<400$ days. ${ }^{15}$

Participants with a negative screening result were invited for the next regular screening round. Those with an indeterminate screening result underwent a low-dose follow-up CT scan to measure volume growth and VDT after 6 weeks to 4 months or after 12 months (depending on nodule volume and screening rounds), to define their definitive screening result (negative or positive). Those with a positive screening result were referred to a pulmonologist for a diagnostic work-up. If no lung cancer was diagnosed, the participant was referred to the next regular screening round. If lung cancer was confirmed, the patient received treatment according to the Dutch National guidelines. Medical data of these patients were collected prospectively.

\section{Definitions}

Current smokers were those who were smoking or who had smoked in the last 7 days before completion of the baseline (risk) questionnaires. Former smokers have quit smoking for 10 years or less. Variables that were calculated were pack-years (20 cigarettes smoked per day for 1 year) and body mass index (BMI) (body weight (in kilograms)/the square of body length (in metres)). New nodules were nodules which were labelled as 'new nodule' or labelled as 'not new, but too small in previous scan to be detectable' by radiologists. ${ }^{24}$

Regular round scans were the first CT examinations in a regular screening round (years 1, 2, 4 and 6.5). Follow-up scans were repeat LDCT scans after an indeterminate result in one of the four regular rounds. A screening-detected lung cancer was defined as a lung cancer diagnosed by a pulmonologist within 12-24 months, depending on the screening round, after referral for a positive screening. Lung cancer detection rate was defined as the number of screening-detected lung cancers divided by the number of screened participants. Regular scan result was defined as the result of the first CT examination in a screening round, while the definitive outcome of the screening (screening result) was made after inclusion of the results of the follow-up scans at the conclusion of that screening round.

\section{Risk groups}

Three unique subgroups were identified based on regular scan results of the first three screening rounds: (1) participants with solely negative results $(\mathrm{n}=3856 ; 73 \%)$; (2) participants with $\geq 1$ indeterminate result and never a positive result $(n=1342$, $25.5 \%)$; and (3) participants with $\geq 1$ positive result $(\mathrm{n}=81$, $1.5 \%$; online supplementary table $\mathrm{S} 1$ ).

Based on the regular scan results of round 3, three other unique subgroups were identified: (1) those with a negative result in round $3(\mathrm{n}=4925,93.5 \%)$; (2) those with an indeterminate result in round $3(\mathrm{n}=324,6.2 \%)$; and (3) those with a positive result in round $3(\mathrm{n}=19,0.4 \%)$. 


\section{Statistical analyses}

None of the continuous variables were distributed normally, tested by using the Kolmogorov-Smirnov test and examining $\mathrm{Q}-\mathrm{Q}$ plots. Therefore, the variables were described by using medians and IQRs. Differences between the continuous variables across the subgroups were calculated by using the median test or analysis of variance, depending on number of subgroups. Differences between nominal variables were calculated by using a $\chi^{2}$ test. Differences between categorical variables were tested by using a Mann-Whitney U test or Kruskal-Wallis test, depending on the number of subgroups. To test differences between the subgroups regarding the probability of having a negative or nonnegative (indeterminate or positive test result) in the fourth round, and to test differences between the subgroups regarding the risk for detecting lung cancer in the fourth round, a logistics regression analysis was used. The associations of gender, packyears smoked, age at randomisation, smoking status at randomisation and age of starting smoking with the screening outcome of the fourth round were assessed through univariate and multivariate analyses. For multivariate analyses, an ordinal logistic regression model was developed using backward selection. In addition, variables were also tested for interactions. For all analyses, a $p$ value $<0.05$ was considered significant, and IBM SPSS Statistics V.21 was used.

\section{RESULTS}

In the first three rounds, 7557, 7295 and 6922 participants were screened, respectively. ${ }^{23}$ In round 4, 5279 participants were screened and 5380 scans were performed; of which 5279 were regular scans and 101 follow-up scans. ${ }^{16}$ In this section, the probabilities of screening outcomes and risk for detecting lung cancer in round 4 were calculated based on the regular scan results alone. An overview of these probabilities based on the definitive screening results of round 4 is presented in the online data supplement (see table S1).

\section{Risk stratification based on the results of the first three screening rounds}

Based on the regular scan results of the first three rounds, three subgroups were identified: (1) participants with only negative results; (2) participants with $\geq 1$ indeterminate, but never a positive result; and (3) participants with $\geq 1$ positive result (table 1). Those from group 1 were slightly younger ( 57.0 vs 58.0 years, $\mathrm{p}<0.001)$ compared with the participants of the other subgroups. Participants with $\geq 1$ positive result had smoked statistically significant slightly more pack-years than the other subgroups $(p=0.02)$. No significant differences were observed between gender and baseline smoking status among the subgroups $(\mathrm{p}=0.66$ and $\mathrm{p}=0.23$, respectively).

Participants with $\geq 1$ indeterminate result, but never a positive result (OR 1.89, $\mathrm{p}=0.001)$, and participants with $\geq 1$ positive result $(3.77, \mathrm{p}<0.001$; table 2 ) had a significantly higher OR of receiving a non-negative scan result (eg, an indeterminate or a positive result) in round 4 compared with the group with solely negative results in the first three rounds (the reference group).

In univariate analysis, only the screening history $(p<0.001)$ and smoked pack-years $(\mathrm{p}=0.01)$ significantly predicted the regular scan result in round 4 , while gender $(p=0.70)$, age $(\mathrm{p}=0.13)$ and baseline smoking status $(\mathrm{p}=0.75)$ did not. In multivariate analysis, screening history and smoked pack-years remained significant predictors $(p<0.001$ and $p=0.02$, respectively): the model suggests that screening history and pack-years are positively associated with the screening outcome in the fourth round (see online supplementary table S4). Interaction between subgroups and smoked pack-years was not significant $(\mathrm{p}=0.89)$.

In round 4, 43 participants were diagnosed with 46 screening-detected lung cancers. OR for detecting lung cancer in round 4 differed between the subgroups as well: relative to the group with only negative results, the group with $\geq 1$ indeterminate result (but never a positive result) had an OR of 2.77 $(\mathrm{p}<0.001)$ for detecting lung cancer in round 4 (table 3$)$. No lung cancer was detected in the group with $\geq 1$ positive result. None of the following factors predicted significantly the detection of lung cancer in round 4: gender $(p=0.71)$, age $(p=0.10)$, starting age of smoking $(p=0.20)$, smoking status $(0.28)$ or pack-years smoked (0.09; all data not shown). Multivariate analysis showed no statistically significance for age and pack-years smoked.

A total of $22(51.2 \%)$ of the participants with screen-detected lung cancer in round 4 had solely negative scan results in the first three screening rounds, and in 20 (90.9\%) of those participants lung cancer was detected in a new nodule (data not shown). The remaining 21 (48.8\%) screen-detected lung cancers in round 4 were detected among participants with at least one indeterminate scan result in the previous three screening rounds, and in 12 (57.1\%) of those participants lung cancer was detected in a new nodule (data not shown).

Participants with only negative regular scan results were stratified by pack-years smoked. Therefore, six categories were made: $<25,26-30,31-35,36-40,41-45$ and $>45$ years. For each category, the risk for lung cancer detection in the fourth round was calculated. The first five categories had a lung cancer risk between $0.2 \%$ (the first category) and $0.7 \%$ (the fifth category). In other words, the risk for detecting lung cancer in the fourth

Table 1 Baseline characteristics of subgroups based on the previous screening rounds and of all participants of the fourth screening round (based on regular scan results)

\begin{tabular}{|c|c|c|c|c|c|}
\hline \multirow[b]{2}{*}{ Baseline characteristics } & \multicolumn{3}{|c|}{ Subgroups based on the results of the previous three screening rounds* } & \multirow{2}{*}{$\begin{array}{l}\text { All participants of the } \\
\text { fourth screening round }\end{array}$} & \multirow[b]{2}{*}{ p Valuet } \\
\hline & All negatives & $\geq 1 \times$ indeterminate, but never a positive result & $\geq 1 \times$ positive & & \\
\hline Male, $\mathrm{n} / \mathrm{N}(\%)$ & $3237 / 3856(83.9)$ & $1129 / 1342(84.1)$ & $71 / 81(87.7)$ & $4437 / 5279(84.1)$ & 0.66 \\
\hline Age, median (IQR) & $57.0(7.0)$ & $58.0(7.0)$ & $58.0(7.0)$ & $58.0(8.0)$ & $<0.001$ \\
\hline Current baseline smokers, $\mathrm{n} / \mathrm{N}(\%)$ & $2086 / 3856(54.1)$ & $753 / 1342(56.1)$ & $39 / 81(48.1)$ & $2878 / 5279(54.5)$ & 0.23 \\
\hline Pack-years at baseline, median (IQR) & $38.0(19.8)$ & $38.0(19.8)$ & $38.7(23.5)$ & $38.0(19.8)$ & 0.02 \\
\hline Total, n (\%) & $3856(73.0)$ & $1342(25.5)$ & $81(1.5)$ & $5279(100.0)$ & - \\
\hline
\end{tabular}


Table 2 Risk calculation for subgroups based on the regular scan results of the first three rounds

\begin{tabular}{|c|c|c|c|c|c|c|c|c|c|c|c|}
\hline & \multirow{2}{*}{\multicolumn{2}{|c|}{ Total group }} & \multicolumn{8}{|c|}{ Regular round scan results of the fourth screening round } & \multirow[b]{3}{*}{ p Value } \\
\hline & & & \multicolumn{2}{|c|}{ Negative } & \multicolumn{2}{|c|}{ Indeterminate } & \multicolumn{2}{|c|}{ Positive } & \multicolumn{2}{|c|}{$\begin{array}{l}\text { Non-negative } \\
\text { result* }\end{array}$} & \\
\hline & $\mathbf{N}$ & Per cent & n & Per cent & n & Per cent & n & Per cent & OR & $95 \% \mathrm{Cl}$ & \\
\hline \multicolumn{12}{|l|}{ Subgroups } \\
\hline All negatives & 3856 & 73.0 & 3747 & 97.2 & 61 & 1.6 & 48 & 1.2 & REF† & REF† & NA \\
\hline$\geq 1 \times$ indeterminate, but never a positive result & 1342 & 25.5 & 1272 & 94.8 & 37 & 2.8 & 33 & 2.5 & 1.9 & 1.4 to 2.6 & 0.001 \\
\hline$\geq 1 \times$ positive result & 81 & 1.5 & 73 & 90.1 & 3 & 3.7 & 5 & 6.2 & 3.8 & 1.8 to 8.0 & $<0.001$ \\
\hline All participants screened in round 4 & 5279 & 100.0 & 5092 & 96.5 & 101 & 1.9 & 86 & 1.6 & - & - & - \\
\hline
\end{tabular}

${ }^{*}$ Non-negative result means an indeterminate or a positive scan result (based on logistic regression).

†The subgroup with only negative scan results in the first three rounds was the reference group to calculate the OR.

Table 3 Risk to detect lung cancer in round 4 for subgroups based on the regular scan results of the first three rounds

\begin{tabular}{|c|c|c|c|c|c|c|c|}
\hline & \multicolumn{6}{|c|}{ Screening-detected lung cancer in round 4} & \multirow[b]{3}{*}{ p Value } \\
\hline & \multicolumn{2}{|c|}{$\begin{array}{l}\text { No screening-detected } \\
\text { lung cancer }\end{array}$} & \multicolumn{2}{|c|}{$\begin{array}{l}\text { Yes, a screening- } \\
\text { detected lung cancer }\end{array}$} & \multirow[b]{2}{*}{$O R^{*}$} & \multirow[b]{2}{*}{$95 \% \mathrm{Cl}$} & \\
\hline & $\mathbf{n}$ & Per cent & $\mathbf{N}$ & Per cent & & & \\
\hline \multicolumn{8}{|l|}{ Subgroups } \\
\hline All negatives & $3834 / 3856$ & 99.4 & $22 / 3856$ & 0.6 & REF† & REF† & NA \\
\hline$\geq 1 \times$ indeterminate, but never a positive result & $1321 / 1342$ & 98.4 & $21 / 1342$ & 1.6 & 2.77 & 1.52 to 5.05 & 0.001 \\
\hline$\geq 1 \times$ positive result & $81 / 81$ & 100.0 & - & - & NA & NA & NA \\
\hline All participants screened in round 4 & $5236 / 5279$ & 99.2 & $43 / 5279$ & 0.8 & - & - & - \\
\hline
\end{tabular}

Table 4 Baseline characteristics of subgroups based on the regular scan results of the third screening round alone

\begin{tabular}{|c|c|c|c|c|}
\hline \multirow[b]{2}{*}{ Baseline characteristics } & \multicolumn{3}{|c|}{ Subgroups based on the screening results of the third round* } & \multirow[b]{2}{*}{ p Value } \\
\hline & Negative & Indeterminate & Positive & \\
\hline Male, n/N (\%) & $4143 / 4925(84.1)$ & 270/324 (83.3) & $16 / 19(84.2)$ & 0.93 \\
\hline Age, median (IQR) & $57.0(8.0)$ & $59.0(8.0)$ & $59.0(7.0)$ & $<0.001$ \\
\hline Current smokers, n/N (\%) & $2672 / 4925(54.3)$ & $195 / 324(60.2)$ & $7 / 19(36.8)$ & 0.04 \\
\hline Pack-years, median (IQR) & $38.0(19.8)$ & $38.7(19.8)$ & $43.2(30.0)$ & 0.05 \\
\hline
\end{tabular}

round for those who smoked less than 45 pack-years was lower than $0.7 \%$. For those who smoked more than 45 pack-years $(n=1091)$, lung cancer detection rate in round 4 was $1.1 \%$ $(p=0.04)$. No correlation was observed between pack-years smoked and lung cancer detection rate in the final round (all data not shown) for the other subgroups.

\section{Risk stratifications based on the previous screening round}

Round 4 was performed 2.5 years after the third screening round and 5.5 years after the baseline scan. Participants with a negative scan result in round 3 were significantly younger at baseline than those with an indeterminate or a positive third round result ( 57.0 years vs 59.0 and 59.0 years, $\mathrm{p}<0.001$; table 4 ). The probability for detecting lung cancer in the fourth round differed between participants with a negative scan result and an indeterminate scan result: $0.6 \%$ vs $3.7 \%$, respectively $(\mathrm{p}<0.001$; tables 5 and 6). No lung cancer was detected in round 4 in the small group with a positive scan result in round 3 .
Participants with a screen-detected lung cancer

Across all four screening rounds, 243 out of 7582 participants were diagnosed with a total of 255 screening-detected lung cancers. Participants with a screening-detected lung cancer were significantly older (61 vs 58 years, $\mathrm{p}<0.001)$ and had smoked more pack-years $(44.0$ vs 38.0 years, $\mathrm{p}<0.001)$ than those without a screening-detected lung cancer. Of those with screendetected lung cancers, $28.4 \%$ had $\geq 1$ indeterminate scan result (but never a positive test result initially) and $71.6 \%$ had $\geq 1$ positive scan result (this group also contains those with once a negative or an indeterminate result) before diagnoses of lung cancer. No differences were seen in gender $(p=0.98)$, baseline smoking status $(p=0.61)$ or in BMI between participants with or without screening-detected lung cancer $(\mathrm{p}=0.38$; all data not shown).

\section{DISCUSSION}

This study demonstrated that individual's screenings history can be used as a risk stratification tool for their further screening 
Table 5 Risk calculation for subgroups based on the regular scan results of round 3 alone

\begin{tabular}{|c|c|c|c|c|c|c|c|c|c|c|c|}
\hline \multirow[b]{3}{*}{ Result round 3} & \multirow{2}{*}{\multicolumn{2}{|c|}{ Total group }} & \multicolumn{8}{|c|}{ Regular round scan results of the fourth screening round } & \multirow[b]{3}{*}{ p Value } \\
\hline & & & \multicolumn{2}{|c|}{ Negative } & \multicolumn{2}{|c|}{ Indeterminate } & \multicolumn{2}{|c|}{ Positive } & \multicolumn{2}{|c|}{ Non-negative result* } & \\
\hline & n & Per cent & $\mathbf{n}$ & Per cent & n & Per cent & n & Per cent & OR & $95 \% \mathrm{Cl}$ & \\
\hline Negative & 4925 & 93.5 & 4770 & 96.9 & 88 & 1.8 & 67 & 1.4 & REFt & REF† & NA \\
\hline Indeterminate & 324 & 6.2 & 293 & 90.4 & 12 & 3.7 & 19 & 5.9 & 3.3 & 2.2 to 4.9 & $<0.001$ \\
\hline Positive & 19 & 0.4 & 18 & 94.7 & 1 & 5.3 & - & - & 1.7 & 0.2 to 12.5 & 0.60 \\
\hline Total & 5268 & 100.0 & 5081 & 96.5 & 101 & 1.9 & 86 & 1.6 & - & - & - \\
\hline
\end{tabular}

*Non-negative result means an indeterminate or a positive scan result.

†The group with a negative scan result in round 3 was the reference group to calculate the OR (based on logistic regression).

Table 6 Risk to detect lung cancer in round 4 for subgroups based on the regular scan results of round 3 alone

\begin{tabular}{|c|c|c|c|c|c|c|c|}
\hline & \multicolumn{6}{|c|}{ Screen-detected lung cancer in round 4} & \multirow[b]{3}{*}{$p$ Value } \\
\hline & \multicolumn{2}{|c|}{$\begin{array}{l}\text { No screening-detected lung } \\
\text { cancer }\end{array}$} & \multicolumn{2}{|c|}{$\begin{array}{l}\text { Yes, a screening- detected } \\
\text { lung cancer }\end{array}$} & \multirow[b]{2}{*}{ OR* } & \multirow[b]{2}{*}{$95 \% \mathrm{Cl}$} & \\
\hline & $\mathbf{n}$ & Per cent & $\mathbf{n}$ & Per cent & & & \\
\hline \multicolumn{8}{|l|}{ Subgroups } \\
\hline Negative & $4894 / 4925$ & 99.4 & $31 / 4925$ & 0.6 & REFt & REF† & \\
\hline Indeterminate & $312 / 324$ & 96.3 & $12 / 324$ & 3.7 & 6.1 & 3.1 to 11.9 & $<0.001$ \\
\hline Positive & $19 / 19$ & 100 & - & - & NA & NA & NA \\
\hline
\end{tabular}

regime. The probability for screening outcome in the fourth round differs across previous screening test result(s). Also, the risk for detecting lung cancer in the fourth screening round differs based on the previous screening outcome(s).

Previous NELSON results showed that the risk for detecting lung cancer in the subsequent second and third rounds differed among the baseline scan result. ${ }^{23}$ Those with an indeterminate or a positive baseline scan result had a higher risk for detecting lung cancer in round 2 or 3, compared with those with a negative baseline scan result. In this study, the results indicated that the probability for non-negative (ie, indeterminate or positive) scan result in the fourth round was higher for those with $\geq 1$ indeterminate (but never a positive result) and those with $\geq 1$ positive result in the first three rounds, compared with those with only negative results in the first three rounds. Furthermore, it was demonstrated that having an older age and have smoked more pack-years smoked were both significant predictors for non-negative result. These results were in line with our previous study results. ${ }^{23}$ None of the lung cancers detected in round 4 was detected in the group with previously $\geq 1$ positive scan result. Moreover, the risk for detecting lung cancer in the final fourth round was non-significantly higher for those with an indeterminate or a positive definitive screening result compared with solely negative screening results (OR of 2.95 and 2.10, respectively).

The combined results of the previous screening rounds turned out to predict the screening outcome (scan results or lung cancer detection risk) in the fourth screening round. The third round test result predicted the fourth round test result after an interval of 2.5 years; for those with a negative third scan, a subsequent round with a 2.5 years interval seemed even short, as the lung cancer risk was $<1 \%$ (as across all screening rounds). ${ }^{1623}$ Moreover, those with previous solely negative scan results and those with a third negative scan result may not need to be screened for more than 2.5 years, as the lung cancer detection rates were $<0.7 \%$ or $1.1 \%$ at most for the fourth round, respectively. However, in almost $90 \%$ of those with solely negative scan results, the lung cancer was detected in a new nodule. Although malignant new nodules might be fast growing, detection at early stage with LDCT seems possible. ${ }^{24}$

Furthermore, it was showed that having an indeterminate scan result gives a higher risk for a non-negative scan result and a higher risk for lung cancer detection in the final round. However, only a minority of the indeterminate nodules turn out to be malignant. With the growing evidence, cut-off points of the nodule management should be evaluated regularly to further optimise the ratio between benefits (eg, mortality reduction) and harms (eg, false positive, unnecessary work-up) of the protocol. Moreover, cancer can evolve from nodules which are not seen before on the scan (eg, from new nodules). One explanation could be 'field cancerisation', in which it is assumed that large areas of the bronchial epithelium are affected by smoking, leading to areas with metaplasia and dysplasia which sometimes turn out to be cancer and sometimes not. Moreover, it is known that in heavy smokers nodules appear and disappear and come up in different pulmonary areas and are not always malignant.

Although only the NELSON trial used increasing screening intervals, the NLST showed in a recent retrospective analysis that participants with a negative baseline result had a lower incidence of lung cancer at baseline as well as a lower lung cancer detection rate in the subsequent rounds $(0.34 \%)$ compared with all screened participants $(1.0 \%) .^{7}$ Furthermore, the lung cancer incidence and mortality for those with solely negative screening results was even lower than for those with a negative baseline screening. Their findings suggested that for the larger part of 
the screened population, it may lead to a better harm-benefit ratio to offer risk-based incidental screening rounds to participants with different screening intervals. In the NELSON trial, end results and cost-effectiveness analyses, and therefore the harm-benefit ratios of screening scenarios, are yet unknown. However, the current study concludes that previous screening history seems to be useful for risk stratification and to refine the screening protocol for subgroups with different risks for lung cancer. ${ }^{23} 2428$

Major strengths of this study are the large-scale, populationbased randomised study design and its volumetric-based nodule management, leading to three initial screening outcomes. However, in this substudy, small numbers of screening-detected lung cancers were found in the fourth round and some subgroups had small numbers of participants. Furthermore, a subselection of screened participants was used: participants should have been screened in one of the three screening rounds as well as in the final screening round. Additionally, almost 1500 participants were lost to follow-up (no actual addresses) in the fourth round, since additional informed consent was required to perform the screening round. ${ }^{16}$

In conclusion, the screening test result(s) might have major implications on the total number of scans needed for those who undergo lung cancer screening. This is useful for the further optimisation of the harm-benefit ratio of a lung cancer screening programme.

\section{Author affiliations}

${ }^{1}$ Department of Public Health, Erasmus Medical Center, University Medical Center Rotterdam, Rotterdam, The Netherlands

${ }^{2}$ Department of Radiology, University Medical Center Utrecht, Utrecht, The Netherlands

${ }^{3}$ University of Groningen, University Medical Center Groningen, Center for Medical Imaging - North East Netherlands, Groningen, The Netherlands

${ }^{4}$ Department of Radiology, Kennemer Gasthuis, Haarlem, The Netherlands

${ }^{5}$ Department of Pulmonary Medicine, KU leuven, University Hospital Leuven, Leuven, Belgium

${ }^{6}$ Department of Pulmonary Diseases, University of Groningen, University Medical Center Groningen, Groningen, The Netherlands

Acknowledgements The authors thank the system controllers R. Faber and F.J.P. Santegoets, and the secretary M.Quak (all from the department of Public Health, Erasmus University Medical Center Rotterdam) for their contribution and maintenance of the database. Furthermore, the authors thank R. Ziengs (University Medical Center Groningen) and S. van Amelsvoort-van der Vorst (University Medical Center Utrecht). Finally, the authors thank the Dutch Cancer Registry (NKR) for the data linkages.

Contributors UY-K, CVdA, MO and HdK: involvement in the conception, hypothesis delineation and design of the study. UY-K, CvdA, PAdJ, ES, $J W, R V, M O$ and HdK: acquisition of the data or the analysis and interpretation of such information. UY-K, CvdA, PAdJ, MH, ES, JW, KN, HG, RV, KtH, MO and HdK: writing the article or substantial involvement in its revision prior to submission.

Funding The NELSON trial is presently supported by 'The Netherlands Organisation for Health Research and Development' (ZonMw).

Competing interests Siemens Germany provided four digital workstations and LungCARE for the performance of 3D measurements of the nodules. Roche diagnostics provided a grant for the performance of proteomics research. The corresponding author had full access to all the data in the study and had final responsibility for the decision to submit for publication.

\section{Patient consent Obtained.}

Ethics approval The NELSON trial was approved by the Dutch Minister of Health and the ethical board at each participating centre. The original NELSON study protocol consisted of three screening rounds. An additional written informed consent form was obtained from all participants who were willing to participate in the fourth screening round.

Provenance and peer review Not commissioned; externally peer reviewed.

\section{REFERENCES}

1 Torre LA, Siegel RL, Jemal A. Lung cancer statistics. Adv Exp Med Biol 2016:893:1-19.

2 van Rens MT, Zanen P, Brutel de La Riviere A, et al. Survival in synchronous vs. single lung cancer: upstaging better reflects prognosis. Chest 2000;118:952-8.

3 Moyer VA, U.S. Preventive Services Task Force. Screening for lung cancer: U.S Preventive Services Task Force recommendation statement. Ann Intern Med 2014:160:330-8

4 Aberle DR, Adams AM, Berg CD, et al. Reduced lung-cancer mortality with low-dose computed tomographic screening. N Engl J Med 2011;365:395-409.

5 Field JK, Duffy SW. Lung cancer CT screening: is annual screening necessary? Lancet Oncol 2016:5:543-4.

6 Horeweg N, Scholten ET, de Jong PA, et al. Detection of lung cancer through low-dose CT screening (NELSON): a prespecified analysis of screening test performance and interval cancers. Lancet Oncol 2014;15:1342-50.

7 Patz EF Jr, Greco E, Gatsonis C, et al. Lung cancer incidence and mortality in National Lung Screening Trial participants who underwent low-dose CT prevalence screening: a retrospective cohort analysis of a randomised, multicentre, diagnostic screening trial. Lancet Oncol 2016:17:590-9.

8 Baldwin DR, Callister ME. What is the optimum screening strategy for the early detection of lung cancer. Clin Oncol 2016;28:672-81.

9 van der Aalst CM, Ten Haaf K, de Koning HJ. Lung cancer screening: latest developments and unanswered questions. Lancet Respir Med 2016:4:749-61.

10 Saghir Z, Dirksen A, Ashraf $\mathrm{H}$, et al. CT screening for lung cancer brings forward early disease. The randomised Danish lung cancer screening trial: status after five annual screening rounds with low-dose CT. Thorax 2012;67:296-301.

11 Wille MM, Dirksen A, Ashraf $\mathrm{H}$, et al. Results of the randomized Danish lung cancer screening trial with focus on high-risk profiling. Am J respire Crit Care Med 2016;193:542-51.

12 Infante $\mathrm{M}$, Cavuto $\mathrm{S}$, Lutman FR, et al. A randomized study of lung cancer screening with spiral computed tomography: three-year results from the DANTE trial. Am J respire Crit Care Med 2009:180:445-53.

13 Pastorino U, Rossi M, Rosato V, et al. Annual or biennial CT screening versus observation in heavy smokers: 5 -year results of the MILD trial. Eur J Cancer Prev 2012;21:308-15.

14 van lersel CA, de Koning HJ, Draisma G, et al. Risk-based selection from the general population in a screening trial: selection criteria, recruitment and power for the Dutch-Belgian randomised lung cancer multi-slice CT screening trial (NELSON) Int J Cancer 2007;120:868-74.

15 van Klaveren RJ, Oudkerk M, Prokop $M$, et al. Management of lung nodules detected by volume CT scanning. N Engl J Med 2009;361:2221-9.

16 Yousaf-Khan $U$, van der Aalst $C$, de Jong PA, et al. Final screening round of the NELSON lung cancer screening trial: the effect of a 2.5 -year screening interval. Thorax 2017;72:48-56.

17 Aberle DR, DeMello S, Berg CD, et al. Results of the two incidence screenings in The National Lung Screening Trial. N Engl J Med 2013;369:920-31.

18 Aberle DR, Berg CD, Black WC, et al. The National Lung Screening Trial: overview and study design. Radiology 2011;258:243-53.

19 Cassidy A, Myles JP, van Tongeren $M$, et al. The LLP risk model: an individual risk prediction model for lung cancer. Br J Cancer 2008;98:270-6.

20 Tammemagi CM, Pinsky PF, Caporaso NE, et al. Lung cancer risk prediction: prostate, lung, colorectal and ovarian cancer screening trial models and validation. J Natl Cancer Inst 2011;103:1058-68.

21 Tammemägi MC, Katki HA, Hocking WG, et al. Selection criteria for lung-cance screening. N Engl J Med 2013;368:728-36.

22 Maisonneuve $\mathrm{P}$, Bagnardi $\mathrm{V}$, Bellomi $\mathrm{M}$, et al. Lung cancer risk prediction to select smokers for screening CT-a model based on the Italian COSMOS trial. Cancer Prev Res 2011:4:1778-89.

23 Horeweg $\mathrm{N}$, van der Aalst CM, Vliegenthart $\mathrm{R}$, et al. Volumetric computed tomography screening for lung cancer: three rounds of the NELSON trial. Eur Respir J 2013:42:1659-67.

24 Walter JE, Heuvelmans MA, de Jong PA, et al. Occurrence and lung cancer probability of new solid nodules at incidence screening with low-dose CT: analysis of data from the randomised, controlled NELSON trial. Lancet Oncol 2016;17:907-16.

$25 \mathrm{Xu}$ DM, Gietema $\mathrm{H}$, de Koning $\mathrm{H}$, et al. Nodule management protocol of the NELSON randomised lung cancer screening trial. Lung Cancer 2006;54:177-84.

26 Wang Y, van Klaveren RJ, van der Zaag-Loonen $\mathrm{HJ}$, et al. Effect of nodule characteristics on variability of semiautomated volume measurements in pulmonary nodules detected in a lung cancer screening program. Radiology 2008;248:625-31.

27 Xie X, Zhao Y, Snijder RA, et al. Sensitivity and accuracy of volumetry of pulmonary nodules on low-dose 16- and 64-row multi-detector CT: an anthropomorphic phantom study. Eur Radiol 2013;23:139-47.

28 Horeweg $\mathrm{N}$, van Rosmalen J, Heuvelmans MA, et al. Lung cancer probability in patients with CT-detected pulmonary nodules: a prespecified analysis of data from the NELSON trial of low-dose CT screening. Lancet Oncol 2014;15:1332-41. 